\title{
Bronchial cartilage in chronic bronchitis
}

\author{
M.K. TANDON AND ALASTAIR H. CAMPBELL \\ From the Central Office, Repatriation Department, St. Kilda Road, South Melbourne, Victoria, Australia
}

\begin{abstract}
An examination has been made of the amount of bronchial cartilage in the subsegmental bronchi of nine chronic bronchitics and seven controls. The axial pathway in four or five representative bronchopulmonary segments were carefully dissected in each lung. In each segment the mean extent of cartilage arranged circumferentially in the walls of the bronchi was significantly less in the chronic bronchitic patient than in the controls. Of the 42 specimens from the various bronchopulmonary segments of the controls, the cartilage was arranged circumferentially to the fifth generation or further in 33 , but extended this far in only 14 of the 50 specimens from the chronic bronchitics. Using certain defined criteria, deficiency of cartilage was found in 25 of 50 specimens from 11 lungs of the chronic bronchitics and in only one of 42 specimens from nine control lungs. There was a negative correlation between the degree of bronchial gland enlargement, as measured by the gland/wall ratio, and the average extent of circumferentially arranged cartilage along the bronchi $(r=-0.8, P<0.01)$. Similarly, the number of bronchopulmonary segments affected increased and the average extent of circumferentially arranged cartilage decreased with increase in severity of the bronchitis. We think that the deficiency of bronchial cartilage in chronic bronchitics would favour collapse of the affected bronchi during forceful expiration or cough.
\end{abstract}

There have been conflicting claims concerning the state of the bronchial cartilage in patients with chronic bronchitis or emphysema. Wright (1960) described a reduction in the number and size of the bronchial cartilaginous plates in patients with chronic bronchitis and emphysema. Smooth muscle and fibrous tissue were also reduced in amount. Deficiency of bronchial cartilage with generalized bronchiectasis has been described in children (Williams and Campbell, 1960). In these cases the deficiency may have been developmental rather than acquired.

In two other investigations, deficiencies of bronchial cartilage in selected bronchi could not be demonstrated. Restrepo and Heard (1964) sectioned the bronchus to the basal segments and the inferior lingular bronchus and did not find any difference in the area of the cartilage in patients with bronchitis and emphysema compared with normals. Similarly, Greenberg, Boushy and Jenkins (1967) measured the width of the cartilage in sections from the main stem, lobar, and segmental bronchi and found no difference between patients with emphysema and those without emphysema.

The discrepancy between the various investigations could be due to examination of different parts of the bronchial tree. Wright (1960) did not describe clearly which of the segmental bronchial generations had defective cartilage, but his observations included bronchi more peripheral than the lobar and first generation segmental bronchi examined by Restrepo and Heard (1964) and Greenberg et al. (1967).

In the present investigation a comparison has been made of the amount of cartilage in the branches of various segmental bronchi of patients with chronic bronchitis and in controls.

\section{METHODS AND MATERIALS}

SUBJECTS The lungs were obtained at necropsy from men who had died at the Repatriation General Hospital, Heidelberg, near Melbourne, Australia. Seven subjects (aged 26-84 years) who had been without symptoms of chronic bronchitis or emphysema during life served as controls and nine lungs were examined from this group.

Nine bronchitic subjects (aged 67-79 years) were selected on the basis of persistent productive cough of several years' duration. In some, the severity of the condition had been assessed by pulmonary function studies. Eleven lungs were examined from this group.

EXAMINATION OF BRONCHI Particular attention was given to detecting all the cartilage in segmental bronchi and their branches. This was achieved by careful dissection of the bronchi and by staining the cartilage with acid toluidine blue. The distances into the bronchial tree were determined in terms of the number of bronchial generations along the bronchial pathway. The generations of the bronchi are the segments of the tubes between successive subdivisions or branches (Hayward and Reid, 1952).

In all counts of bronchial generations the segmental 
bronchi were counted as the first generation. Each of the two bronchi produced by the first division of the segmental bronchus was regarded as belonging to the second generation. The four bronchi produced by the division of each of the two bronchi of the second generation belong to the third generation, and so on.

Each lung was fixed by intrabronchial injection of $8 \%$ formol saline at a pressure of $30 \mathrm{~cm} . \mathrm{H}_{2} \mathrm{O}$ for at least one week.

Axial pathways in five representative bronchopulmonary segments (apical segment of the lower lobe, both segments of middle or lingular lobes, lateral and posterior segments of the lower lobe) were dissected in each lung. In several lungs the presence of fibrosis or other factors reduced the number of satisfactory dissections to four segments. When the axial bronchus divided dichotomously, both branching bronchi were traced.

The dissected bronchial pathways were then drawn and the generations carefully indicated on the draw- ing. The bronchi were then opened and stitched to aO wire mesh ready for staining; a second sketch was made of the opened bronchi.

The staining procedure was based upon that des-市 cribed by Stovin (1959). The bronchi were washed in running tap water to remove the formalin. Suturedes to a chrome-plated wire mesh, they were immersed $\vec{\circ}$ for 16-24 hours in toluidine blue solution having $\mathrm{a}-$ $p \mathrm{H}$ between 1 and 2 . By immersion in 70\% alcohol $\vec{\omega}$ for about half an hour the toluidine blue was removed? from tissues other than the cartilage. The specimen was then dehydrated by immersion in absolute alco-i hol two or three times depending upon thickness of the wall. The bronchi were then placed in cedar wood: oil for four or five days until the non-cartilaginous? tissues became transparent. The specimen was then 0 placed in xylol in a Petri dish.

Generally, well-stained specimens were obtained with this procedure. In one case, inspection showed $\mathbb{D}$ uneven staining of the cartilage. Satisfactory staining
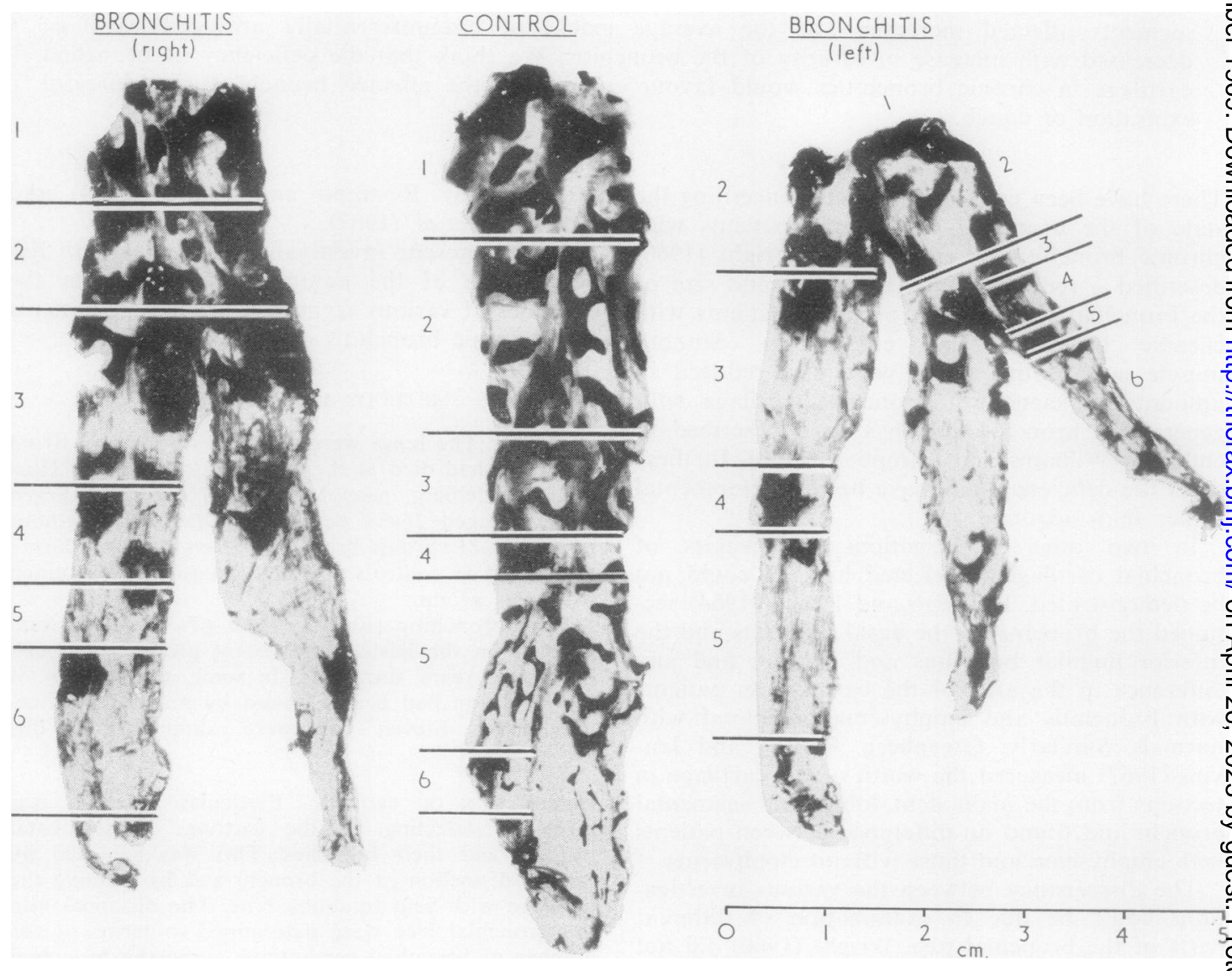

FIG. 1. Posterior basal segments from a control and from both lungs of a bronchitic subject. In the control, circum ferentially arranged cartilage extends up to the 6th generation, whereas in the bronchitic it is seen up to the $3 r d \bar{D}$ generation (right) and in the left up to the 5th generation in one axial branch and to the 2 nd generation in another branch 
was obtained by repeating the process. In another case, with poor and uneven staining, microscopic examination showed non-staining of the cartilage. This case was excluded.

In all other cases the staining appeared even and adequate.

The presence or absence of cartilage observed macroscopically in the well-stained specimens was confirmed by microscopic examination of sections of 24 bronchial generations from four subjects (two controls and two bronchitics).

With the help of the original drawings indicating the bronchial generations, the level at which the bronchial cartilage ceased to be arrayed circumferentially was noted for each bronchopulmonary segment. The specimens were also examined for variation in the size and distribution of the cartilaginous plates. All the specimens were photographed to obtain a permanent record.
From sections of the main lobar bronchi, the gland/wall ratio (Reid, 1960) was measured in all seven of the control group, and ranged between 0.26 and 0.42 (average 0.34 ). In the bronohitic group it was measured in six and ranged between 0.45 to 0.61 (average 0.52 ).

It was not possible to assess the degree of emphysema present in the lungs examined, as dissection of the bronchopulmonary segments destroyed the parenchyma.

\section{RESULTS}

The main difference between the chronic bronchitics and the controls was shown by determining the distance along the axial pathways that cartilage was arranged circumferentially. The cartilage was regarded as being arranged circumferentially when

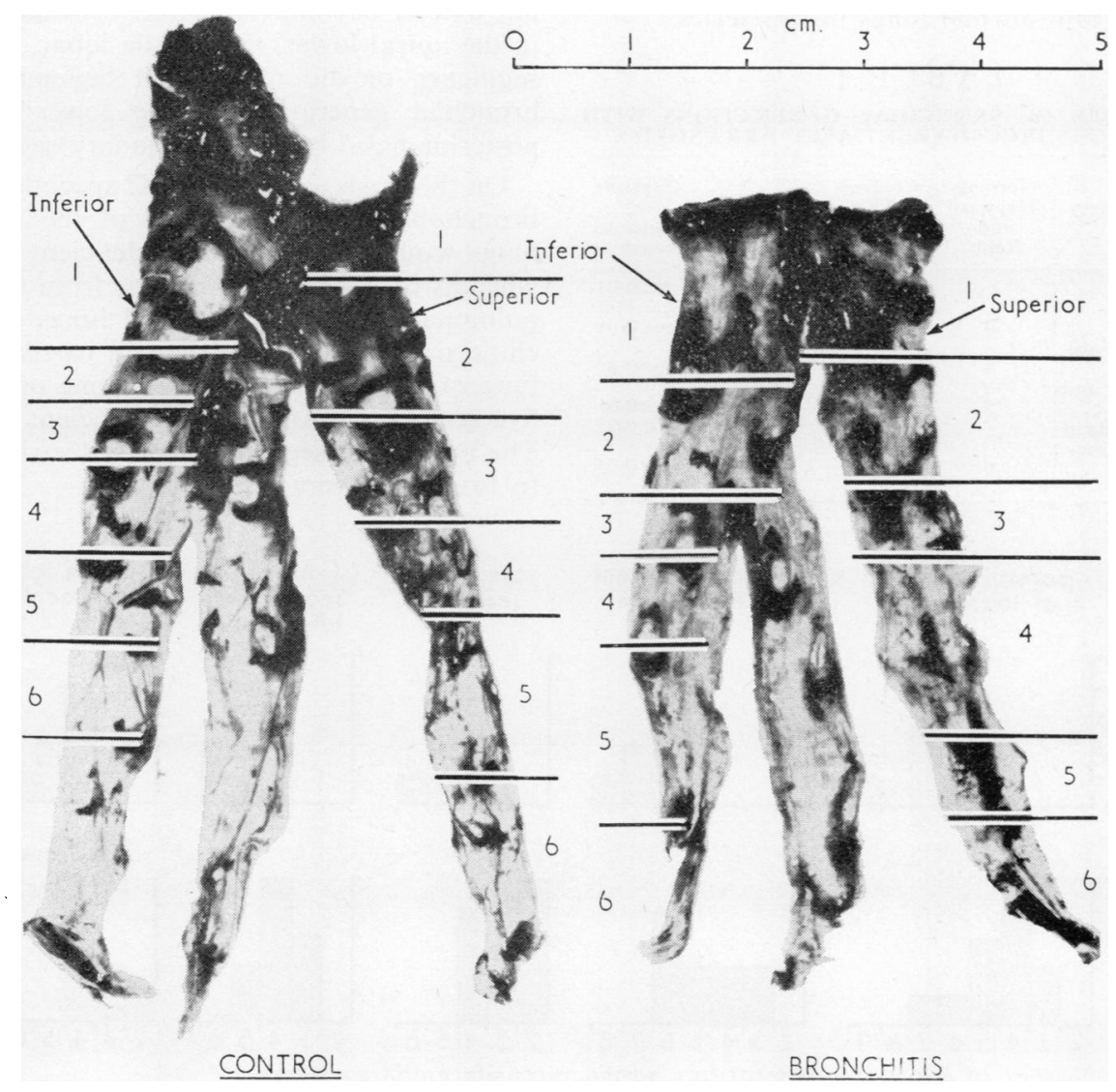

FIG. 2. Lingular lobar segments of a control and a bronchitic case. Circumferentially arranged cartilage in the superior and inferior segments of the control is seen up to 4th and 5th generations respectively, whereas in the bronchitic it is seen up to the 3rd generation in the superior and to the 2nd generation in the inferior segment. 
pieces were scattered around the tube on all sides.

Examination of the segmental bronchi for several generations revealed that there was less bronchial cartilage in the bronchitic subjects than in the controls. The deficiency was less apparent in the first or second bronchial generations and affected the more distal generations to a varying degree (Figs 1 and 2).

In all the bronchopulmonary segments examined, the mean extent of the circumferential arrangement of cartilage along the axial pathways was significantly less extensive in the chronic bronchitic patients than in the controls (Table I). The average extent of circumferential cartilage in certain segments in normal subjects studied by Hayward and Reid (1952) have been included for comparison. Considering the difference in technique, their results compare reasonably closely with those from normal lungs in this series.

\section{T A B L E I}

MEAN NUMBER OF BRONCHIAL GENERATIONS WITH CARTILAGE CIRCUMFERENTIALLY ARRANGED

\begin{tabular}{l|c|c|c|c}
\hline $\begin{array}{c}\text { Bronchopulmonary } \\
\text { Segments }\end{array}$ & $\begin{array}{c}\text { Normals } \\
\text { (Hayward } \\
\text { and } \\
\text { Reid) }\end{array}$ & $\begin{array}{c}\text { Controls } \\
\text { (This } \\
\text { Investiga- } \\
\text { tion) }\end{array}$ & $\begin{array}{c}\text { Chronic } \\
\text { Bronchitic } \\
\text { Subjects }\end{array}$ & $\begin{array}{c}\text { Controls } \\
\text { v. } \\
\text { Chronic } \\
\text { Bronchitics }\end{array}$ \\
\hline $\begin{array}{c}\text { All segments } \\
\text { Apical segment_ } \\
\text { lower lobe }\end{array}$ & 4 & 5.4 & 4.0 & $P<0.01$ \\
$\begin{array}{c}\text { Middle lobe (lateral } \\
\text { and medial) } \ldots\end{array}$ & 4 & 4.8 & 3.9 & $\mathbf{P}<0.01$ \\
$\begin{array}{c}\text { Lingula (superior and } \\
\text { inferior) }\end{array}$ & 5 & 4.4 & 3.5 & $\mathbf{P}<0.05$ \\
$\begin{array}{c}\text { Lateral basal segment } \\
\text { Posterior basal seg- } \\
\text { ment }\end{array}$ & 6 & 6.4 & 4.6 & $\mathbf{P}<0.01$ \\
\hline
\end{tabular}

In the 42 specimens from the bronchopulmonaryc segments of the controls, the cartilage waso arranged circumferentially to the fifth generation or further in 33 but extended this far in only $14 \frac{1}{2}$ of the 50 specimens from the bronchopulmonaryon segments of the chronic bronchitics. The extent of the circumferential arrangement of cartilage in terms of bronchial generations has been displayed for each of the bronchopulmonary segmentse examined (Fig. 3). Frequently in the bronchitics $\vec{x}$ the cartilage was arranged circumferentially for an relatively small number of bronchial generations.

ชั

NUMBER OF SEGMENTS INVOLVED IN INDIVIDUALO CASES For the purpose of assessing the number. of bronchopulmonary segments with deficients cartilage in each lung, the cartilage was considere $\mathbb{B}$ deficient when the circumferential cartilage did的 not extend beyond the third bronchial generation in the apical lower, the middle lobar, and lingular segments, or did not extend beyond the fourth $\overrightarrow{0}$ bronchial generation in the lower lateral ande posterior basal bronchopulmonary segments.

On this basis, only one of 42 specimens from thes bronchopulmonary segments of the nine controp lungs would be regarded as deficient in cartilage whereas 25 of the 50 specimens from the broncho-D pulmonary segments of the 11 lungs of the bron chitic patients were deficient in cartilage. In each? lung of the bronchitics at least one of the four or five segments examined was deficient in cartilage The number of segments involved varied from one to four and averaged 2.3.

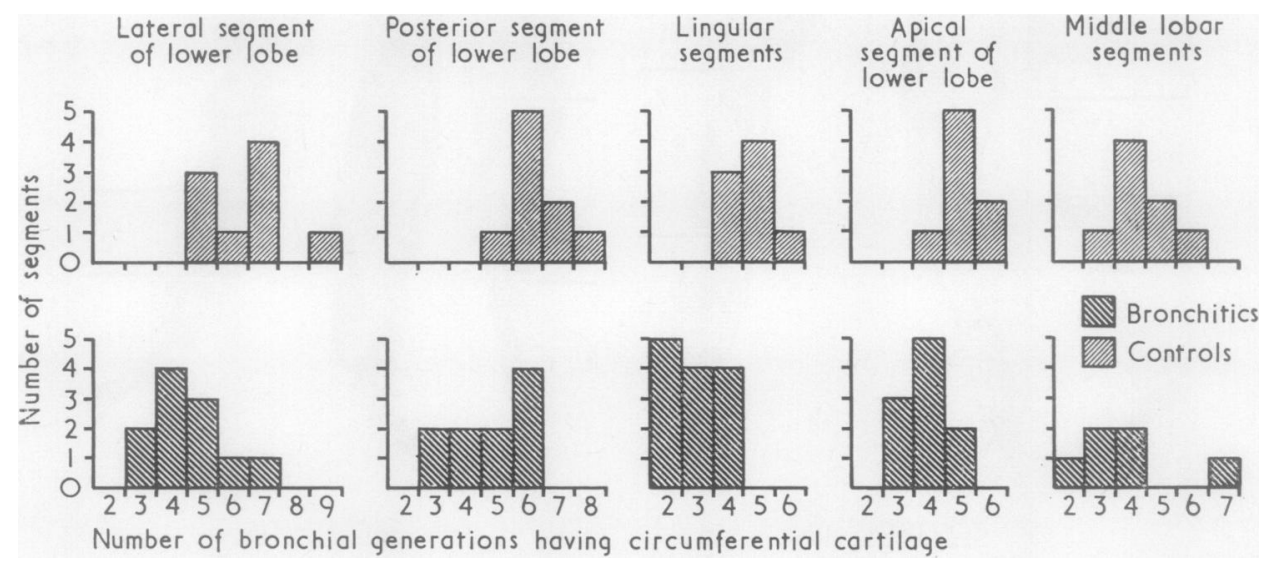

FIG. 3. Comparative distribution of number of segments according to number of bronchial generations having circumferential cartilage in the different bronchopulmonary segments of the controls and the tronchitics. 
DEGREE OF DEFICIENCY OF CARTILAGE AND SEVERITY OF BRONCHITIS In controls and bronchitics in whom the bronchial gland/wall ratio had been measured, and in whom the bronchial cartilage had been examined in five bronchopulmonary segments, a comparison was made between the deficiency of bronchial cartilage and the degree of hypertrophy of the bronchial mucous glands.

As shown in Fig. 4, there is a negative correlation between the gland/wall ratio and the average extent of the circumferentially arranged cartilage along the axial pathways; the greater the hypertrophy of the glands, the more deficient the bronchial cartilage $(\mathrm{r}=-0.85, \mathrm{P}<0.01)$.

Four of the bronchitic patients had been shown to have severe airway obstruction during life. The forced expiratory volume/vital capacity ratio was less than $20 \%$ in all four. All these patients had had bronchitis for more than 10 years. The other five bronchitic patients had not had respiratory function tests and were judged to have moderately severe bronchitis on clinical grounds; the duration of bronchitis had been less than 10 years in all five.

In each of these two groups of bronchitics and in the controls, the average number of segments per lung regarded as being deficient in bronchial cartilage and the average number of bronchial

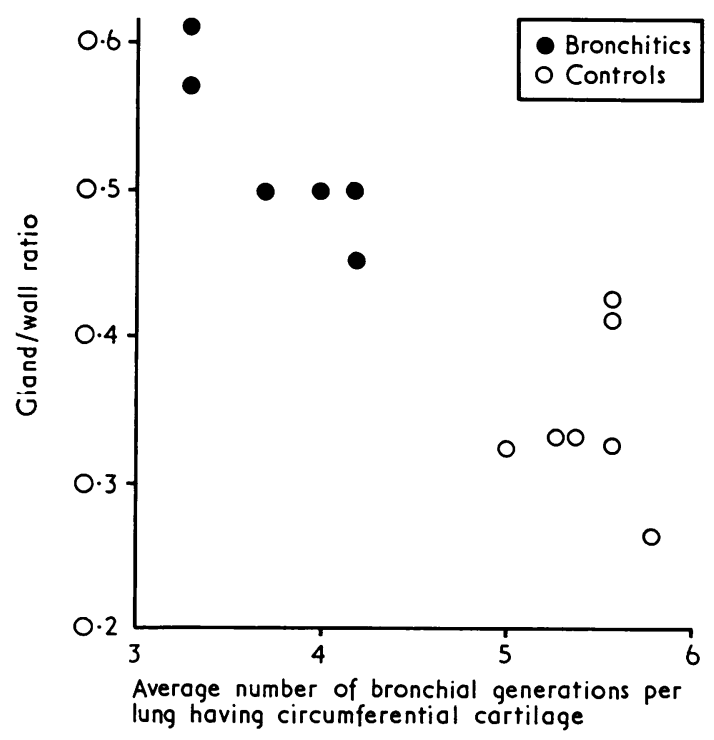

FIG. 4. The gland/wall ratio, studied in six bronchitic and seven control cases, showed a negative correlation with the average number of bronchial generations per lung having circumferential cartilage $(r=-0.85, \mathrm{P}<0.01)$. generations with circumferentially arranged cartilage have been compared. It is shown in Table II that the number of bronchopulmonary segments affected increased with the severity of the bronchitis, whereas the average extent of circumferentially arranged cartilage decreased with severity of the bronchitis.

\section{T A B L E I I}

RELATIONSHIP BETWEEN SEVERITY OF BRONCHITIS AND DEFICIENCY OF CARTILAGE

\begin{tabular}{|c|c|c|}
\hline & $\begin{array}{l}\text { Mean No. of } \\
\text { Segments, per } \\
\text { Lung, Deficient } \\
\text { in Cartilage }\end{array}$ & $\begin{array}{l}\text { Mean No. of Bronchial } \\
\text { Generations with } \\
\text { Circumferentially } \\
\text { Arranged Cartilage }\end{array}$ \\
\hline $\begin{array}{l}\text { Severe bronchitis } \\
\text { Moderately severe bron- }\end{array}$ & 3.0 & $3 \cdot 7$ \\
\hline 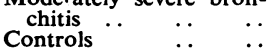 & $\begin{array}{l}2 \cdot 0 \\
0 \cdot 1\end{array}$ & $\begin{array}{l}4 \cdot 1 \\
5 \cdot 4\end{array}$ \\
\hline
\end{tabular}

The significance of these relationships was tested after calculation of correlation coefficients. When the grade of bronchitis is compared with the mean number of segments with deficient cartilage in each with the mean number of segments with deficient cartilage in each lung, $r=0.85, P<0.01$. Comparing the grade of bronchitis with the
mean number of bronchial generations with circumferentially arranged mean number of bronchial generations with circumferentially arranged
cartilage in the bronchopulmonary segments examined, $r=-0.87$, $\mathbf{P}<0.01$.

\section{DISCUSSION}

Restrepo and Heard (1964) and Greenberg et al. (1967) failed to demonstrate deficiency of cartilage in selected bronchi from patients with bronchitis or emphysema. The bronchi examined by these investigators were first generation segmental or larger bronchi. In the present investigation, the deficiency of cartilage was apparent in the bronchi smaller than the first segmental generation.

The walls of these bronchi, for several generations along axial pathways, normally receive support from the circumferentially arranged cartilage to which is attached a fibroelastic network. When these bronchi have been dissected free, palpation reveals that for a distance into the segment of about five divisions the tubes feel relatively rigid and resist occlusion by external pressure. Distal to this point they are readily compresed (Hayward and Reid, 1952).

During a forced expiration, the pressure in the alveoli surrounding the bronchial tubes is raised and is greater than the intraluminal pressure; thus the transmural pressure is positive and tends to compress the bronchial tube. The degree of compression depends in part upon the compliance of the bronchial wall. It can be safely assumed that bronchi which are normally supported by circumferential cartilage will become more compliant if the cartilage becomes deficient. It is likely that such bronchi will tend to collapse during forced expiration, considerably obstructing air flow through the affected bronchi. 
Collapse of subsegmental bronchi has been observed during life in patients with obstructive airway disease (Rayl, 1965 ; Gayrard and Charpin, 1968). Also a dynamic bronchographic study of post-mortem human lungs (Petty, Miercort, Ryan, Vincent, Filley, and Mitchell, 1965) has shown that premature and excessive airway collapse occurred in the intraparenchymal airways in patients with severe symptoms of airway obstruction. Excessive and premature airway collapse was not observed in normal lungs or in most lungs with mild emphysema. Moderate to severe emphysema with or without chronic bronchitis was present in the lungs with the most premature collapse. Lungs in which chronic bronchitis was the predominant lesion also demonstrated varying degrees of airway collapse. As a 'working hypothesis', Petty et al. (1965) suggested that when sufficient destruction of the alveolar tissue occurs, small and medium sized intraparenchymal airways lose the radial traction forces that normally maintain the patency of the conducting airways and that this process allows the premature and excessive bronchial collapse observed.

That loss of radial traction on the bronchi was not the sole cause of the premature airway collapse can be suggested as Petty et al. (1965) observed that in one lung with very severe emphysema premature airway collapse did not occur; and in some patients with bronchitis, but without severe emphysema, premature airway collapse occurred.

Irrespective of the role of diminished radial traction on bronchial calibre, the findings of the present investigation suggest that, at least in part, the premature airway collapse of the intrasegmental bronchi observed by Petty et al. (1965) could have been contributed to by weakening of the bronchial walls through partial deficiency of the circumferentially arranged cartilage. ${ }^{1}$

The cause of the deficiency of the bronchial cartilage requires further investigation. Cartilage deficiency associated with bronchiectasis has been observed by Williams and Campbell (1960) in children, and is probably a developmental abnormality. It is possible that minor grades of such developmental deficiency of bronchial cartilage might persist into adult life.

However, all the men studied in the present investigation had been healthy in their youth and

ISee Addendum fit for war service. They developed bronchitis 용

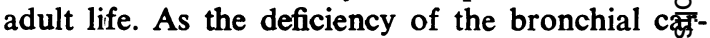
tilage is related to the severity of the chroris bronchitis, as judged by the glandular enlargement in the bronchial wall and clinical severity, it seems likely that in these patients the deficiency of cardlage has been acquired and may be due to atrophy following repeated infection of the bronchis: walls. There was no indication that age led to atrophy of the cartilage as all the controls, except one, were over 50 years of age and the amount ff cartilage present compared closely with that ofer served in normals by Hayward and Reid (1952)

We wish to thank Dr. Peter Campbell for helpfự advice, Dr. D. G. Buist for considerable assistance $\overrightarrow{\mathrm{in}}$ developing the technique, and the Chairman of the Repatriation Commission for permission to publiq this paper.

\section{ADDENDUM}

Since completion of this paper, an article Maisel, Silvers, Mitchell, and Petty (1968) has been published dealing with causes of dynamic expir tory airway collapse. They reported that at sit of bronchial collapse absence or marked atroplo of cartilage, together with variable loss of bas ment membrane, mucous glands, vessels, smoot muscle and collagen, were significant findings.

\section{REFERENCES}

Gayrard, P., and Charpin, J. (1968). Evaluation of the role of large bronchi in the genesis of air obstruction in normal subject and in various diseases. Amer. Rev. resp. Dis, 97, 1076.

Greenberg, S. D., Boushy, S. F., and Jenkins, D. E. (1967). Chronic bronchitis and emphysema; correlation of pathologic findings Ibid., 96, 918 .

Hayward, J., and Reid, L. McA. (1952). The cartilage of the intra pulmonary bronchi in normal lungs, in bronchiectasis, and massive collapse. Thorax, 7,98 .

Maisel, J. C., Silvers, G. W., Mitchell, R. S., and Petty, T. L. Bronchial atrophy and dynamic expiratory collapse. Amer. Re resp. Dis., $98,988$.

Petty, T. L., Miercort, R., Ryan, S., Vincent, T., Filley, G. F., an Mitchell, R. S. (1965). The functional and bronchographi⿺ evaluation of postmortem human lungs. Ibid., 92, 450 Rayl, J. E. (1965). Tracheobronchial collapse during cough. Radiolog
85, 87.

Reid, L. (1960). Measurement of the bronchial mucous gland layer a diagnostic yardstick in chronic bronchitis. Thorax, 15, 132 .

Restrepo, G. L., and Heard, B. E. (1964). Air trapping in chronic bronchitis and emphysema. Amer. Rev. resp. Dis., 90, 395.

Stovin, P. G. I. (1959). Congenital lobar emphysema. Thorax, 14, 25\%

Williams, H., and Campbell, P. (1960). Generalized bronchiectas associated with deficiency of cartilage in the bronchial tree. Arch
Dis. Childh., 35, 182 .

Wright, R. R. (1960). Bronchial atrophy and collapse in chronic ${ }^{+}$ obstructive pulmonary emphysema. Amer. J. Path., 37, 63 . 